\title{
Satisfactory optimization design for fractional order PID controller
}

\author{
Tianyu Liu ${ }^{\mathrm{a}}$, Yuanjun Jia ${ }^{\mathrm{a}}$, Weidong Jin ${ }^{\mathrm{a}}$, Yong Wang ${ }^{\mathrm{b}, *}$ \\ ${ }^{a}$ Department of Automation, University of Science and Technology of China, Hefei 230022, \\ China \\ ${ }^{b}$ College of Electrical Engineering, Southwest Jiaotong University, Chengdu 610031, China
}

\begin{abstract}
This paper presents a new parameters optimization approach for fractional order PID controllers, which uses a satisfactory optimization model. To fulfill different design performance specifications and constrains of systems, the application of multi-criterion satisfactory optimization to fractional control systems is considered. At the same time, the performance of fractional control systems controlled by fractional order controller and integer order controller is discussed. The simulation illustrates the effectiveness of the proposed method and the superiority of the fractional order controller in both time domain and frequency domain.
\end{abstract}

Keywords: Satisfactory optimization, Multi-criterion satisfactory optimization, Fractional order system, Fractional order controller

\section{Introduction}

The key of optimization in a control system is to solve harmonious between constrains of system and performance specifications. The traditional optimization tries to satisfy optimization targets under constrains. In order to obtain 5 the optimal solution, it is necessary to find an accurate mathematic model and an ideal objective function. Actually, due to lots of complex elements, the actual control systems cannot meet it, even there is no optimal solution for some

\footnotetext{
* Corresponding author

Email address: yongwang@ustc.edu.cn (Yong Wang)
}

Preprint submitted to 2019 ASME IDETC/CIE Conference

May 2, 2019 
optimization problems. For this reason, satisfactory control and satisfactory optimization [1] is considered. Multi-criterion satisfactory optimization model is proposed by Jin et al. in [1]. Then it is employed in signal processing [1, 2], computer network design [3] and fuzzy controller design [4].

In recent years, due to that many systems can be described accurately with the introduction of fractional order calculus, fractional order systems have attracted much attention in the engineering and physics fields, such as supercapacitor [5], human body circuit models [6]. Besides, fractional systems play an increasingly important role in many scientific and engineering problems, such as fractional order system identification [7], stability analysis [8, 9], adaptive control [10, 11], signal processing [12, 13], etc.

The fractional order PID (FOPID) controller is the extension of the inte20 ger order PID (IOPID) controller [14]. Compared to the IOPID controller, the FOPID has two more parameters: the integral order $\lambda$ and the derivative order $\mu$. This make it more flexible. It can exert better robustness and accuracy for different controlled systems. It also can be used in industrial control [15, 16]. However, it is complicated to tune PID parameters. The performance specifications in frequency domain, such as phase margin, gain crossover frequency and robustness to variations in the gain of the plant, are often used to optimize the controller parameters in [16-19]. However, those methods are frequency-based methods. In practice, those methods guarantee good system frequency response but poor time domain response. Two sets of tuning rules for FOPID based on the first Ziegler-Nichols tuning rules are presented in [20]. However, this method in [20] is only valid for those plants, whose step response is S-shaped.

Motivated by the above discussions, a novel method based on multi-criterion satisfactory optimization (MSO) is presented in this paper. The main contributions are concluded as follows: a new fractional PID parameter tuning method 35 is investigated. The MSO method is utilized to tuning controller parameters for the first time. By selecting the appropriate performance specification and satisfactory rate function. This method can guarantee both good time domain performance and frequency domain performance. Several simulations verify 
the effectiveness of this method. In this paper, a

The rest of this paper is organized as follows. Section 2 reviews the fundamental definitions of fractional order calculus and introduces the concept of the FOPID. A novel tuning method proposed for fractional order controllers (FOCs) based on multi-criterion satisfactory optimization model is described in Section 3. In Section 4, several numerical examples are provided. Finally,

45 Section 5 draws some conclusions.

\section{Preliminaries}

The fractional order system is a mathematical model based on the fractional order differential equation. It is important to realize that the words "fractional order systems" mean just "systems which are better described by fractional order mathematical models [14]."

\subsection{Fractional order calculus}

Fractional order calculus means that the orders of the integral and derivative are arbitrary order. Fractional order calculus is an extension of the traditional integer order calculus. In this section, a brief summary of mathematical background about fractional order calculus is introduced. More details can be found in $[21]$.

Generally, the fundamental operator of fractional order calculus can be defined as

$$
{ }_{a} \mathscr{D}_{t}^{\alpha} f(t)= \begin{cases}\frac{\mathrm{d}^{\alpha} \mathrm{f}(\mathrm{t})}{\mathrm{d} t^{\alpha}} & , \operatorname{Re}(\alpha)>0, \\ 1 & , \operatorname{Re}(\alpha)=0, \\ \int_{a}^{t} f(\tau)(\mathrm{d} \tau)^{-\alpha} & , \operatorname{Re}(\alpha)<0,\end{cases}
$$

where $a$ and $t$ are the limits of the operation. $\alpha$ is the order of the integral or derivative, which can be real or complex.

The definitions of fractional order calculus given by different mathematicians are also different. The most popular definitions of fractional order calculus 
mainly have the Riemann-Liouville ( $\mathrm{R}-\mathrm{L}$ ) definition, the Grünwald-Letnikov ( $\mathrm{G}-\mathrm{L})$ definition and the Caputo definition, etc.

The Riemann-Liouville derivative definition of the order $\alpha$ can be given by

$$
{ }_{a}^{\mathrm{R}} \mathscr{D}_{t}^{\alpha} f(t)=\frac{1}{\Gamma(m-a)}\left(\frac{\mathrm{d}}{\mathrm{d} t}\right)^{m} \int_{a}^{t} \frac{f(\tau)}{(t-\tau)^{\alpha-m+1}} \mathrm{~d} \tau,
$$

where $m-1<\alpha<m, m \in \mathbb{N}^{+}, \Gamma(x)=\int_{0}^{\infty} \mathrm{e}^{-t} t^{x-1} \mathrm{~d} t$ is the Gamma function.

The Grünwald-Letnikov definition can be written as

$$
{ }_{a}^{\mathrm{G}} \mathscr{D}_{t}^{\alpha} f(t)=\lim _{h \rightarrow 0} \frac{1}{h^{\alpha}} \sum_{j=0}^{\left\lfloor\frac{t-a}{h}\right\rfloor}(-1)^{j}\left(\begin{array}{c}
\alpha \\
j
\end{array}\right) f(t-j h),
$$

where $h$ is the sample time, $\lfloor\cdot\rfloor$ is the flooring function.

The Caputo derivative definition can be described as

$$
{ }_{a}^{\mathrm{C}} \mathscr{D}_{t}^{\alpha} f(t)=\frac{1}{\Gamma(m-\alpha)} \int_{a}^{t} \frac{f^{(m)}(\tau)}{(t-\tau)^{\alpha-m+1}} \mathrm{~d} \tau,
$$

where $m-1<\alpha<m, m \in \mathbb{N}^{+}$.

Actually the fractional order definitions have no influence on this paper, so those can be abbreviated as ${ }_{a} \mathscr{D}_{t}^{\alpha}$ uniformly. For convenience, the Laplace transform also can be used to solve the differential equation. If we define $F(s)$ as the Laplace transform of the function $f(t), F(s) \equiv \mathscr{L}[f(t)]$. Consider the zero initial conditions, the Laplace transform of fractional order calculus is

$$
\mathscr{L}\left[\mathscr{D}^{\alpha} f(x)\right]=s^{\alpha} F(s),
$$

where $\mathscr{D}^{\alpha}$ represents the $\alpha$ th derivative of $f(t)$ from start point 0 to $t$.

\subsection{Fractional order PID controllers}

The differential equation of the FOPID controller is given (See [14])

$$
u(t)=K_{p} e(t)+K_{i} \mathscr{D}^{-\lambda} e(t)+K_{d} \mathscr{D}^{\mu} e(t),
$$

whose transfer function can be written as

$$
G_{c}(s)=K_{p}+K_{i} s^{-\lambda}+K_{d} s^{\mu} .
$$


As is shown in Fig. 1, for the IOPID controller, the range of orders is limited to four discrete points, corresponding to the P, PI, PD and PID four control model. The FOPID controller extends the four points to the plane defined by selecting the values of $0<\lambda, \mu<2$. This makes it more flexible. It can exert better robustness and accuracy for different controlled systems.

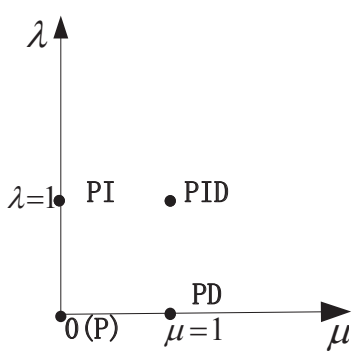

(a) Integer order controller

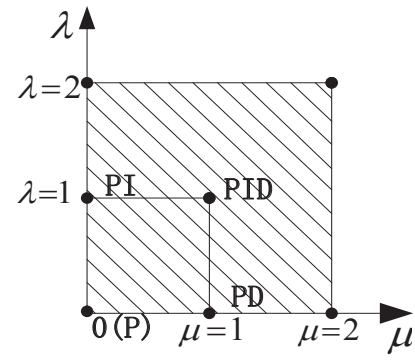

(b) Fractional order controller

Fig. 1. Range of control points of PID controllers with fractional order and integer order

\section{Satisfactory optimization method}

\subsection{Multi-criterion satisfactory optimization model}

In this subsection, the multi-criterion satisfactory optimization model [1], will be introduced .

Suppose the number of parameter variables in the optimization problem is

90 $n$. Let $X$ be the parameter variable set. Then

$$
X=\left\{\left(x_{1}, x_{2}, \ldots, x_{n}\right) \mid x_{i} \in \mathbb{R}, i=1,2, \ldots, n\right\},
$$

where $X \in \mathbb{R}^{n}$.

Assume $m$ is the number of the performance specifications to evaluate system performance, $Q$ is the set of the system performance specifications, we have

$$
Q=\left\{\left(q_{1}, q_{2}, \ldots, q_{m}\right) \mid q_{i} \in \mathbb{R}, k=1,2, \ldots, m\right\}
$$


In the feasible solution space of the closed-loop system, choose a vector of

95 of system performance specification as $q^{j}=\left[q_{1}^{j}, q_{2}^{j}, \ldots, q_{m}^{j}\right]$, where $q_{k}^{j}$ is the performance value of the $k$ th performance specification $q_{k}$ respect to the system feasible solution $x^{j}$. In general, in the actual system the value of performance specification is related to more than one parameter variable. Thus, we have 100 $q_{k}=\varphi(x)$, such that

$$
q=\left[q_{1}, q_{2}, \ldots, q_{m}\right]=\left[\varphi_{1}(x), \varphi_{2}(x), \ldots, \varphi_{m}(x)\right]=\varphi(x),
$$

where $k=1,2, \ldots, m$.

Let $g_{k}: \mathbb{R} \rightarrow[0,1]$ be the satisfactory rate function respect to $q_{k}$, then the satisfactory rate of $q_{k}$ can be described as

$$
s_{k}=g_{k}\left(q_{k}\right)
$$

Denote $s=\left[s_{1}, s_{2}, \ldots, s_{m}\right]$ as the vector of satisfactory rate functions, where ${ }_{105} s \in[0,1]^{m}$. Then, we have

$$
s=g(q)=\left[g_{1}\left(q_{1}\right), g_{2}\left(q_{2}\right), \ldots, g_{m}\left(q_{m}\right)\right]
$$

The synthesis satisfactory rate function is defined as $f:[0,1]^{m} \rightarrow[0,1]$, then

$$
s w=f(s)=f\left(s_{1}, s_{2}, \ldots, s_{m}\right)
$$

where $s w \in[0,1]$.

The value of system synthesis satisfactory function $s w$ represents the synthesis satisfactory rate of this system to the designer under the feasible solution.

In summary, based on the multi-criterion satisfactory optimization model, the optimization problem can be expressed as

$$
\begin{array}{ll}
\max & f(x) \\
\text { s.t. } & \mathrm{g}_{\mathrm{i}}\left(\mathrm{q}_{\mathrm{i}}\right) \in[0,1], \quad \mathrm{i}=1,2,3, \cdots, \mathrm{m},
\end{array}
$$

where $x \in X \subseteq \mathbb{R}^{n}, q \in Q \subseteq \mathbb{R}^{m}$. 


\subsection{Design of the FOPID controller}

In this subsection, a new fractional PID parameter tuning method is investigated. Using the aforementioned method, satisfactory optimization design for the FOPID controller is presented for the first time. The MSO method is mainly used to find the five parameters of the FOPID controller, such that the controlled system obtains a satisfactory performance. The procedures to tune the FOPID are as follows:

step 1 Choosing the parameter variable set. For the FOPID controller, we choose five controller parameters $K_{p}, K_{i}, K_{d}, \lambda$ and $\mu$ as parameter variable set, such that

$$
X=\left[x_{1}, x_{2}, x_{3}, x_{4}, x_{5}\right]=\left[K_{p}, K_{i}, K_{d}, \lambda, \mu\right] .
$$

step 2 Choosing the performance specification set. In this study, three performance specifications in the time domain are concerned. The performance specification set includes overshoot $\sigma \%$, settling time $t_{s}(2 \%)$ and rising time $t_{r}$, such that

$$
Q=\left[q_{1}, q_{2}, q_{3}\right]=\left[\sigma \%, t_{s}, t_{r}\right] .
$$

step 3 Selecting satisfactory rate function of each performance specification.

According to the selected performance specifications, the satisfactory rate functions are designed as

$$
\left[s_{1}, s_{2}, s_{3}\right]=\left[g_{1}(\sigma \%), g_{2}\left(t_{s}\right), g_{3}\left(t_{r}\right)\right] .
$$

step 4 Selecting synthesis satisfactory rate function. In general, the synthesis satisfactory rate function is defined as

$$
s w=\sum_{k=1}^{m} w_{k} s_{k} .
$$

130

The weight reflects the importance of each performance specification, which satisfy $\sum_{k=1}^{m} w_{k}=1$. 
Remark 1. This model provides a framework for parameter optimization, the specific parameter search algorithm can be selected by designer properly such as genetic algorithm, particle swarm optimization algorithm [22].

Remark 2. The satisfactory rate function reflects the tolerance of designer to the variable range of the controller parameters. According to a satisfactory rate function, the optimal problem can be converted to a satisfactory optimization problem. It also influences the convergence of a algorithm [1].

\section{Illustrative examples}

Consider a second-order plant (see [20]), whose transfer function is

$$
G_{1}(s)=\frac{1}{4.32 s^{2}+19.1801 s+1} .
$$

Using the multi-criterion satisfactory optimization model to optimize the FOPID controller. The three satisfactory rate functions $s_{1}, s_{2}$ and $s_{3}$ in this study are shown in Fig. 2. Take, for example, the overshoot $\sigma \%$, the explicit satisfactory rate function is as follow:

$$
s_{1}= \begin{cases}1 & , q_{1} \leq 0, \\ 1-0.01 q_{1} & , 0<q_{1} \leq 10, \\ 1.2-0.03 q_{1} & , 10<q_{1} \leq 40, \\ 0 & , 40<q_{1} .\end{cases}
$$

For simplicity, each performance specification has the same weight. Thus we have

$$
s w=\sum_{k=1}^{m} w_{k} s_{k}, w_{m}=\frac{1}{3}, m=3 .
$$

Genetic algorithm is concerned to search satisfactory parameters. Set the population size $M=80$, the evolution iteration $G=100$, the crossover rate $P_{c}=0.8$, the mutation rate $P_{m}=0.05$. Select the synthesis satisfactory rate $s w$ as fitness, set simulation time to 20 seconds, the sampling interval is 0.01 


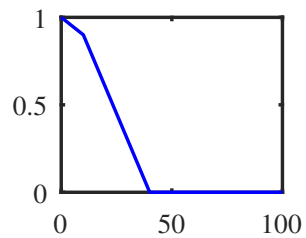

(a) $\sigma(\%)$

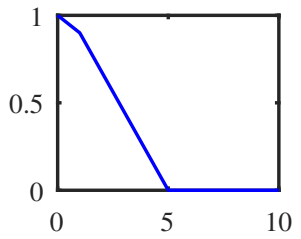

(b) $t_{s}(2 \%)$

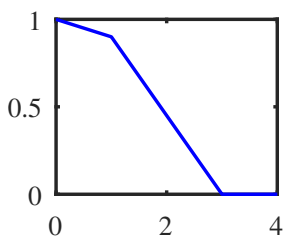

(c) $t_{r}$

Fig. 2. Satisfactory rate functions

seconds. After 100 times iterations, the convergence of the synthesis satisfactory rate is shown in Fig. 3. Then the optimization parameters are shown in Table.

1.

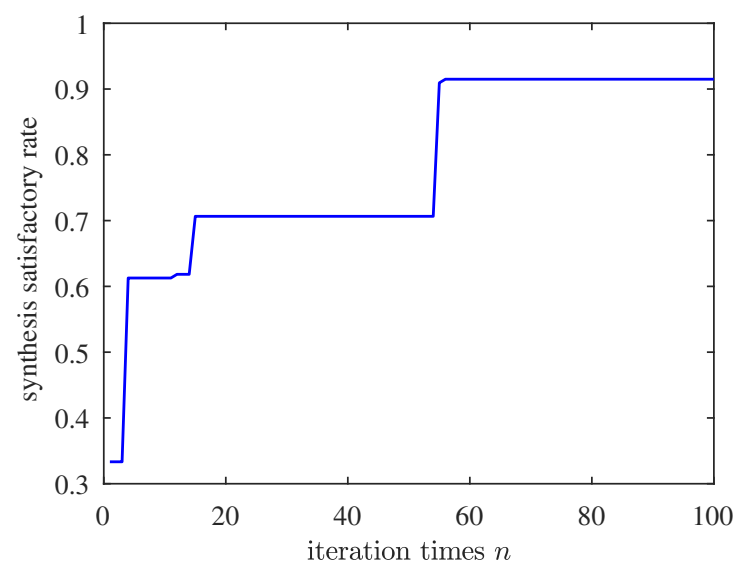

Fig. 3. The convergence of the synthesis satisfactory rate

The corresponding FOPID can be designed as

$$
C_{11}(s)=199.8045+\frac{48.9247}{s^{0.0029}}+48.2893 s^{0.9282} .
$$

Literature [20] presents two sets of tuning rules for fractional PID. The two rules for tuning the parameters of fractional PIDs assumes the plant to have an S-shaped unit-step response. The first rule needs two tables of parameters, while the second, good for a narrower interval of values of $\mathrm{L}$ only, needs only 
Table 1: FOPID controller parameters

\begin{tabular}{lllll}
\hline Parameters & MSO & Ref. [20] $C_{12}$ & Ref. [20] $C_{13}$ & DE \\
\hline$K_{p}$ & 199.8045 & 0.0880 & 6.9928 & 201.6255 \\
$K_{i}$ & 48.9247 & 6.5185 & 12.4044 & 63.9625 \\
$K_{d}$ & 48.2893 & 2.5881 & 4.1066 & 26.9022 \\
$\lambda$ & 0.0029 & 0.6751 & 0.6000 & 0.0498 \\
$\mu$ & 0.9282 & 0.6957 & 0.7805 & 0.6988 \\
\hline
\end{tabular}

160

one. Controllers obtained with the two sets of tuning rules [20] are

$$
\begin{gathered}
C_{12}(s)=0.0880+\frac{6.5185}{s^{0.6751}}+2.5881 s^{0.6957}, \\
C_{13}(s)=6.9928+\frac{12.4044}{s^{0.6000}}+4.1066 s^{0.7805} .
\end{gathered}
$$

The controller obtained with differential evolution (DE) algorithm with parameter self-adjusting [23] is

$$
C_{14}(s)=201.6255+\frac{63.9625}{s^{0.0498}}+26.9022 s^{0.6988} .
$$

In this work, the Oustaloup continuous approximation have been used to approximate fractional order operators to an integer transfer function. The unit-step response, ISE and Bode diagrams of the controlled system $G_{1}(s)$ with different FOCs are shown in Figs. 4, 5 and 6.

This comparison demonstrates that systems with a FOPID controller designed by the MSO method has smaller overshoot, faster response and larger phase margin. Using the proposed MSO method, the system performs faster response in time domain and superiority in the frequency domain.

The specific performances of the controlled system $G_{1}(s)$ with different controllers are shown in Table 2. Compare to other controllers, the controlled system with a controller designed by the MSO method performs a better performance in both time domain and frequency domain, the overall performance of the proposed method is better. The results demonstrate the effectiveness 


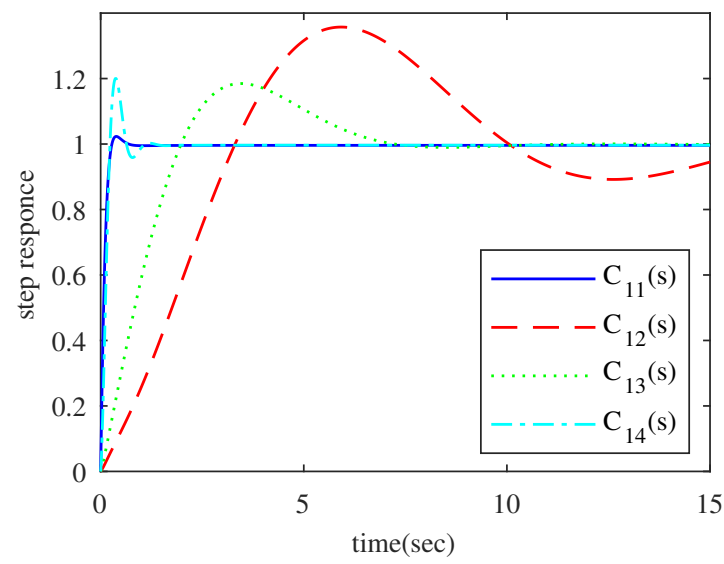

Fig. 4. Step response of $G_{1}(s)$ controlled by different FOCs

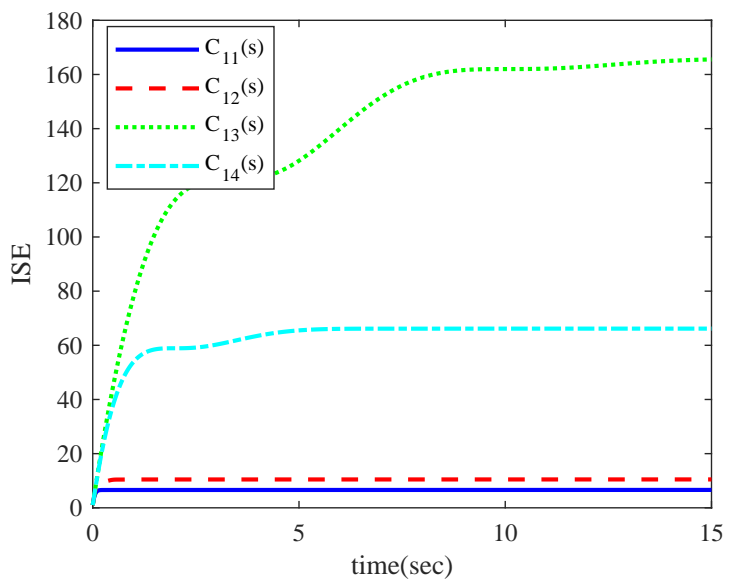

Fig. 5. ISE of $G_{1}(s)$ controlled by different FOCs 

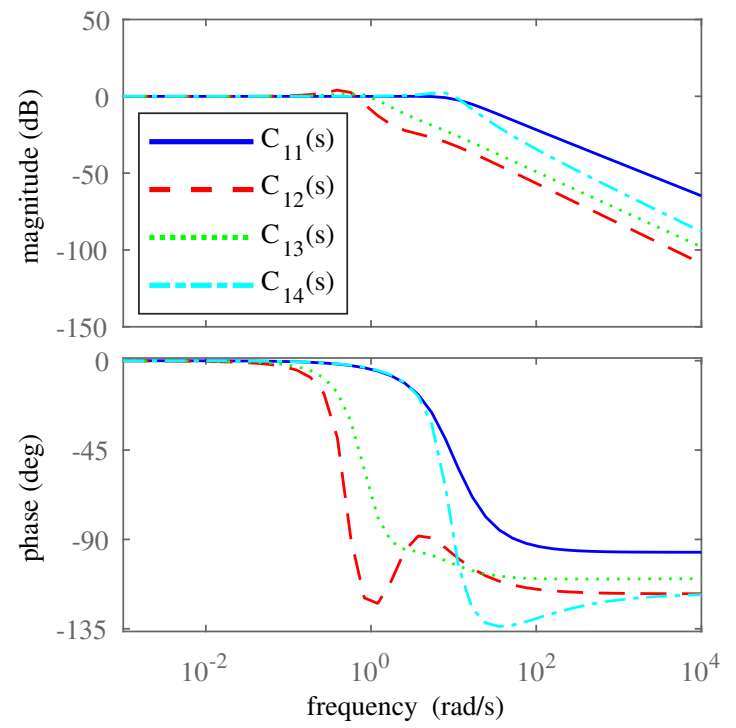

Fig. 6. Bode diagrams of $G_{1}(s)$ controlled by different FOCs

of the proposed method in the parameter optimization design of the fractional order control system.

Table 2: Performance analysis of the controlled system $G_{1}(s)$ under different controllers

\begin{tabular}{lllll}
\hline Controller & $\sigma(\%)$ & $t_{r}(s)$ & $t_{s}(s)$ & $\gamma(\circ)$ \\
\hline$C_{11}(s)$ & 2.3209 & 0.2000 & 0.2200 & 122.5 \\
$C_{12}(s)$ & 35.8 & 3.303 & 15.87 & 69 \\
$C_{13}(s)$ & 18.6 & 1.982 & 6.889 & 99.7 \\
$C_{14}(s)$ & 8.5 & 0.327 & 1.471 & 71.5 \\
\hline
\end{tabular}

\subsection{Fractional order plants}

Consider a fractional order plant [14], whose transfer function is

$$
G_{2}(s)=\frac{1}{0.8 s^{\alpha}+0.5 s^{\beta}+1},
$$


where the system nominal parameter values are $\alpha=2.2$ and $\beta=0.9$. The FOC and integer order controller (IOC) designed by the proposed method, respectively, are

$$
\begin{gathered}
C_{21}(s)=51.3196+\frac{41.0557}{s^{0.0958}}+96.4809 s^{1.8162}, \\
C_{22}(s)=47.1261+\frac{0.7331}{s}+69.0225 s .
\end{gathered}
$$

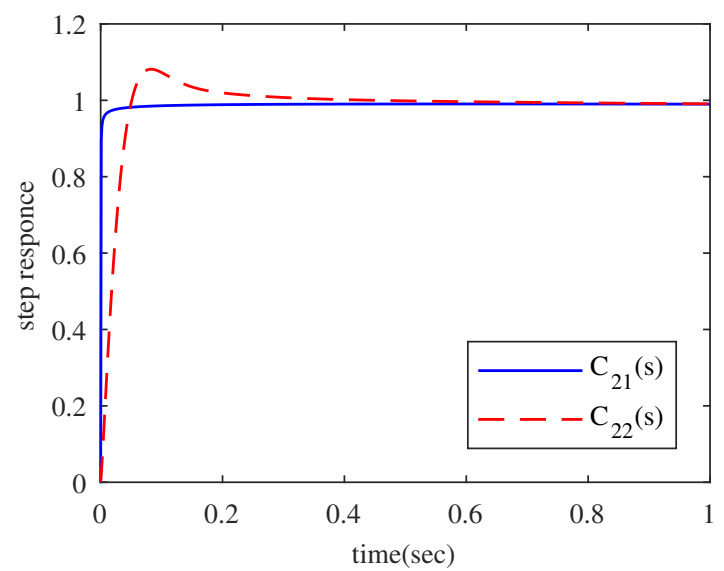

Fig. 7. Step response of $G_{2}(s)$ controlled by the FOC and the IOC

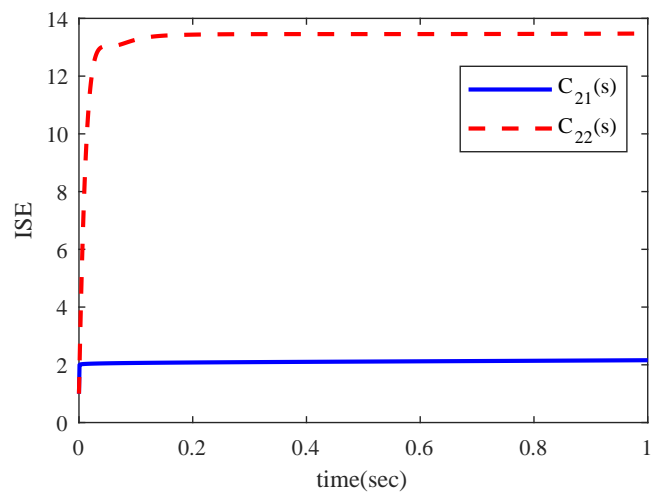

Fig. 8. ISE of $G_{2}(s)$ controlled by the FOC and IOC

The unit-step response and ISE of the controlled system $G_{2}(s)$ with different 
controllers are shown in Fig. 7. Due to the external environment conditions, true system parameters may change. Here, we assume that the disturbances occur in the parameters: $\alpha \in[1.9,2.5], \beta \in[0.7,1.1]$. For the actual system, PID parameters are not the best match parameters. Using the aforementioned controllers, the unit-step response of the disturbed system $G_{2}(s)$ is shown in Fig. 9. Obviously, the performance specifications, such as overshoot, settling time and rising time, are slightly worse, but it still shows good robustness.
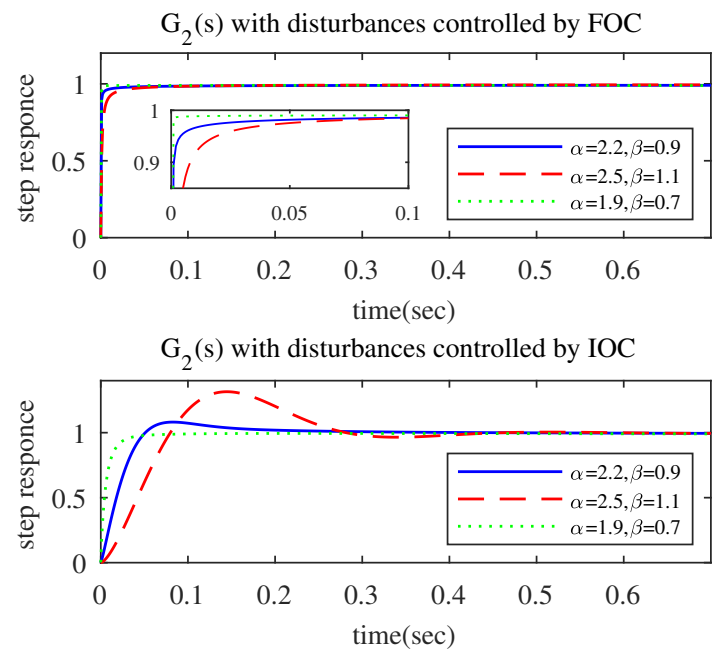

Fig. 9. Step response of $G_{2}(s)$ with disturbances

For fractional order plants, comparing with the IOC, the closed-loop system with the FOC can obtain a better performance, even the disturbances occur in the parameters. It is clear that the FOC can improve the system response by choosing the orders of the integral and derivative flexibly.

\subsection{First-order plants with delay}

Consider a first-order plant with delay [20], whose transfer function is

$$
G_{3}(s)=\frac{k}{1+1.5 s} \mathrm{e}^{-0.1 s} .
$$


Consider the nominal value of $k=1$, controllers obtained with first set of tuning rules and second set of tuning rules [20], respectively, are

$$
\begin{aligned}
& C_{31}(s)=0.6021+\frac{0.6187}{s^{1.3646}}+0.3105 s^{1.0618}, \\
& C_{32}(s)=1.4098+\frac{1.6486}{s^{1.1011}}-0.2139 s^{0.1855} .
\end{aligned}
$$

The FOC and the IOC designed by the proposed method, respectively, are

$$
\begin{gathered}
C_{M S O-F}(s)=0.3715+\frac{6.7253}{s^{0.9404}}+6.9599 s^{0.1251} \\
C_{M S O-I}(s)=6.4516+\frac{2.6393}{s}+0.0782 s
\end{gathered}
$$

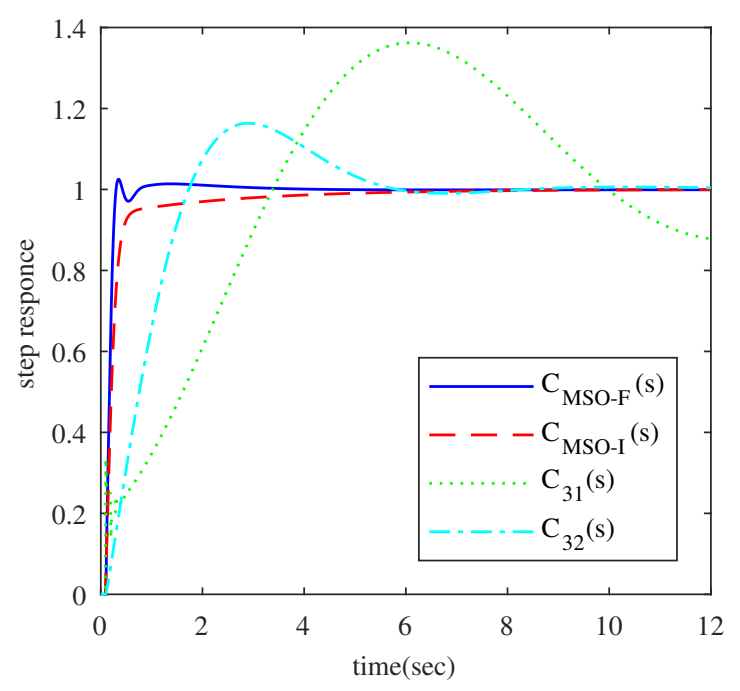

Fig. 10. Step response of $G_{2}(s)$ controlled by different FOCs

205

The unit-step response of the controlled system $G_{2}(s)$ with different controllers, where $k=1$, is shown in Fig. 10.

Assume the value of $k$ has slightly changed, we set the $k=1,1 / 2,1 / 4,1 / 8,1 / 16$ respectively. The unit-step response of system $G_{2}(s)$ controlled by different controllers when $k$ changes is shown in Fig. 11. 
As is shown in Fig. 11, compare to the IOC, the system with the FOC performs a better time domain response. Compared to other methods, the controlled system designed by multi-criterion satisfactory optimization model has a better time domain response. Comparison of Figs. 11(a), 11(b) and 11(c), when the value of $k$ changes, systems with the FOPID controller designed by the MSO method remains smaller overshoot and faster response. By comparing Figs. 11(c) and 11(d), when the value of $k$ changes, the system with the FOPID controller designed by the MSO method remains small over-shoot, but not as the curve of Fig 11(d), holding a short rising time and settling time.

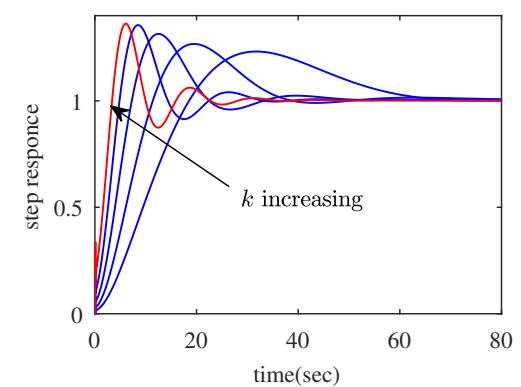

(a) The FOC designed by first set of rules

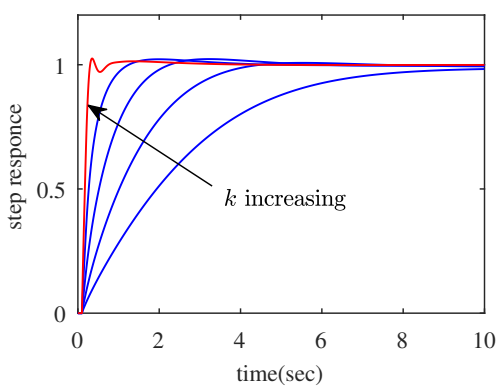

(c) The FOC designed by the MSO

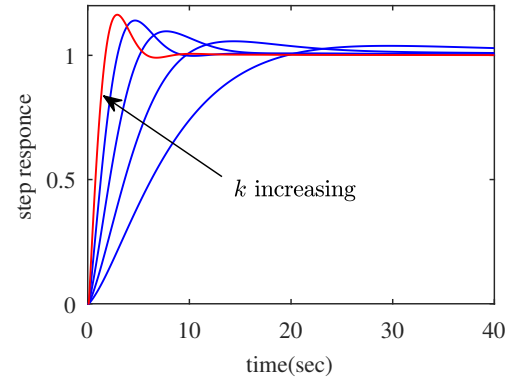

(b) The FOC designed by second set of rules

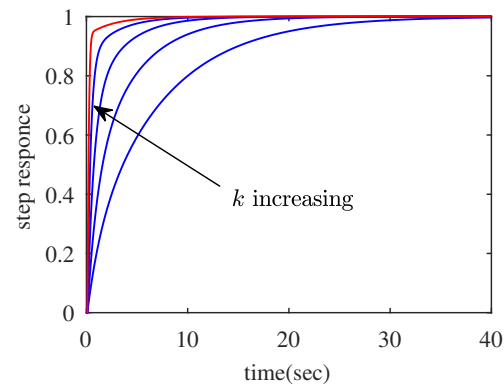

(d) The IOC designed by the MSO

Fig. 11. Step response of $C_{2}(s)$ controlled with PID controllers designed by different methods, when $k$ is $1,1 / 2,1 / 4,1 / 8,1 / 16$.

In conclusion, compare to the IOPID controller, systems controlled by the 
FOPID controller performs a faster and more accurate time domain response; compare to other methods, fractional order systems designed by the MSO method performs a faster and more accurate time domain response.

\section{Conclusions}

A novel method for the FOPID controller parameters setting is presented. Contraposing the complexity of tuning the FOPID controller parameters, a new design guide based on multi-criterion satisfactory optimization model for the FOPID controller is proposed. The results demonstrate the effectiveness of the MSO method. Comparing with the conventional integer order PID controller, frequency domain.

\section{Acknowledgments}

The work described in this paper was fully supported by the National Natural Science Foundation of China (No. 61573332, No. 61601431), the Fundamental Research Funds for the Central Universities (No. WK2100100028), the Anhui Provincial Natural Science Foundation (No. 1708085QF141) and the General Financial Grant from the China Postdoctoral Science Foundation (No. 2016M602032).

\section{References}

${ }_{240}^{241]}$ W. Jin, D. Zhao, G. Li, The application of multi-criterion satisfactory optimization in FIR digital filter design, in: Proceedings of 2000 International Workshop on Autonomous Decentralized Systems, 2000, pp. 227-230. doi:10.1109/IWADS.2000.880912.

[2] G. Zhang, W. Jin, F. Jin, Multi-criterion satisfactory optimization method for designing IIR digital filters, in: Proceedings of 2003 International 
Conference on Communication Technology, 2003, pp. 1484-1490. doi: 10.1109/ICCT . 2003.1209809.

[3] X. Tan, W. Jin, D. Zhao, The application of multi-criterion satisfactory optimization in computer networks design, in: Proceedings of the Fourth International Conference on Parallel and Distributed Computing, Applications and Technologies, 2003, pp. 660-664. doi:10.1109/PDCAT.2003. 1236386.

[4] D. Zhao, W. Jin, The application of multi-criterion satisfactory optimization in fuzzy controller design, in: The 2nd International Workshop on Autonomous Decentralized Systems, 2002, pp. 162-167. doi: 10.1109/IWADS. 2002.1194666.

[5] A. S. Elwakil, A. Allagui, B. J. Maundy, C. Psychalinos, A low frequency oscillator using a super-capacitor, International Journal of Electronics and Communications 70 (7) (2016) 970-973. doi:10.1016/j.aeue.2016.03. 020.

[6] V. D. Santis, V. Martynyuk, A. Lampasi, M. Fedula, M. Ortigueira, Fractional-order circuit models of the human body impedance for compliance tests against contact currents, International Journal of Electronics and Communications 78 (2017) 238-244. doi:10.1016/j.aeue.2017.04.035.

[7] Y. S. Hu, Y. Fan, Y. H. Wei, Y. Wang, Q. Liang, Subspace-based continuous-time identification of fractional order systems from nonuniformly sampled data, International Journal of Systems Science 47 (1) (2016) 122-134. doi:10.1080/00207721.2015.1029568.

[8] Y. H. Wei, Y. Q. Chen, S. S. Cheng, Y. Wang, Completeness on the stability criterion of fractional order LTI systems, Fractional Calculus and Applied Analysis 20 (1) (2017) 159-172. doi:10.1515/fca-2017-0008.

[9] J. G. Lu, Y. Q. Chen, Robust stability and stabilization of fractionalorder interval systems with the fractional order $\alpha$ : The $0<\alpha<1$ case, 
IEEE Transactions on Automatic Control 55 (1) (2010) 152-158. doi: 10.1109/TAC. 2009. 2033738.

[10] Y. Q. Chen, Y. H. Wei, S. Liang, Y. Wang, Indirect model reference adaptive control for a class of fractional order systems, Communications in Nonlinear Science and Numerical Simulation 39 (2016) 458-471. doi:10.1016/j.cnsns.2016.03.016.

[11] Y. H. Wei, P. W. Tse, Y. Zhao, Y. Wang, Adaptive backstepping output feedback control for a class of nonlinear fractional order systems, Nonlinear Dynamics 86 (2) (2016) 1047-1056. doi:10.1007/s11071-016-2945-4.

[12] S. S. Cheng, Y. H. Wei, Y. Q. Chen, S. Liang, Y. Wang, A universal modified LMS algorithm with iteration order hybrid switching, ISA Transactions 67 (2017) 67-75. doi:10.1016/j.isatra.2016.11.019.

[13] S. S. Cheng, Y. H. Wei, Y. Q. Chen, Y. Li, Y. Wang, An innovative fractional order LMS based on variable initial value and gradient order, Signal Processing 133 (2017) 260-269. doi:10.1016/j.sigpro.2016.11.026.

[14] I. Podlubny, Fractional-order systems and $\mathrm{PI}^{\lambda} \mathrm{D}^{\mu}$ controllers, IEEE Transactions on Automatic Control 44 (1) (1999) 208-214. doi:10.1109/9. 739144.

[15] I. Dimeas, I. Petráš, C. Psychalinos, New analog implementation technique for fractional-order controller: a DC motor control, International Journal of Electronics and Communications 78 (2017) 192-200. doi:10.1016/j . aeue.2017.03.010.

[16] C. A. Monje, B. M. Vinagre, V. Feliu, Y. Q. Chen, Tuning and auto-tuning of fractional order controllers for industry applications, Control Engineering Practice 16 (7) (2008) 798-812. doi:10.1016/j.conengprac.2007.08. 006.

[17] H. S. Li, Y. Luo, Y. Q. Chen, A fractional order proportional and derivative (FOPD) motion controller: tuning rule and experiments, IEEE Trans- 
actions on Control Systems Technology 18 (2) (2010) 516-520. doi: 10.1109/TCST . 2009.2019120.

[18] C. A. Monje, B. M. Vinagre, Y. Q. Chen, V. Feliu, On fractional $\mathrm{PI}^{\lambda}$ controllers: some tuning rules for robustness to plant uncertainties, Nonlinear Dynamics 38 (1) (2004) 369-381. doi:10.1007/s11071-004-3767-3.

[19] D. Y. Xue, C. N. Zhao, Fractional order PID controller design for fractional order system, Control Theory and Applications 24 (5) (2007) 771-776. doi:10.3969/j.issn.1000-8152 .2007.05.015.

[20] D. Valério, J. S. D. Costa, Tuning of fractional PID controllers with ZieglerNichols-type rules, Signal Processing 86 (10) (2006) 2771-2784. doi:10. 1016/j.sigpro.2006.02.020.

[21] I. Podlubny, Fractional Differential Equations: an Introduction to Fractional Derivatives, Fractional Differential Equations, to Methods of Their Solution and Some of Their Applications, Academic Press, San Diego, 1999.

[22] M. Mitchell, An Introduction to Genetic Algorithms, MIT press, Massachusetts, 1998.

[23] L. L. Huang, X. L. Zhou, J. H. Xiang, Self-adjusting design on parameters of the fractional order PID controller, Systems Engineering and Electronics 35 (5) (2013) 1064-1069. doi:10.3969/j .issn.1001-506X . 2013.05. 28. 